\title{
Impact of cancer on use of glucose-lowering drug treatment in individuals with diabetes: potential mechanisms
}

\author{
Frans Pouwer
}

Received: 11 February 2015 / Accepted: 20 February 2015 /Published online: 13 March 2015

(C) Springer-Verlag Berlin Heidelberg 2015

Keywords Adherence - Behavioural aspects · Cancer · Comment $\cdot$ Co-morbidity $\cdot$ Compliance $\cdot$ Depression $\cdot$ Diabetes mellitus $\cdot$ Mechanisms · Therapy

\section{Abbreviation \\ GLD Glucose-lowering drugs}

To the Editor: In a very interesting and relevant study, based on the very large dataset from the Eindhoven Cancer Registry, Zanders et al investigated the potential impact of cancer on adherence to glucose-lowering drugs (GLDs; oral medication and/or insulin) in people with diabetes [1]. Their results showed a significant drop in the medication possession ratio, which was used as an indicator for medication adherence. The authors conclude that they found 'a clear decline in adherence to GLD treatment regimens following a cancer diagnosis'. They state that the reason for this decline needs to be further elucidated.

In this letter, I would like to describe two potential mechanisms that were not discussed by Zanders et al in their paper [1]. First, weight loss might have played an important role. Weight loss is common among people with cancer; it is often the first noticeable sign of the disease, but change in appetite and weight loss can also result from anti-cancer therapies. On www.cancer.net, a website of the American Society of Clinical Oncology, it is stated that as many as $40 \%$ of people with cancer report unexplained weight loss at the time of

F. Pouwer $(\bowtie)$

Department of Medical and Clinical Psychology, CoRPS, Tilburg University, Postbus 90153, 5000 LE Tilburg, the Netherlands

e-mail: f.pouwer@tilburguniversity.edu diagnosis [2]. Moreover, up to $80 \%$ of people with advanced cancer experience weight loss and cachexia, the combination of both weight loss and muscle mass loss. Dietary energy restriction and subsequent weight loss can lead to strong improvements of both beta cell function and insulin sensitivity in type 2 diabetes [3] and thus to a decreased need for glucose-lowering drugs. Zanders et al stated that the largest drops in medication use were observed in their study in patients with gastrointestinal or pulmonary cancer and in patients with advanced cancer [1]. Interestingly, Nicolini et al summarised that anorexia, cachexia and malnutrition are particularly common in patients with gastrointestinal cancer, lung cancer and advanced cancer [4]. Weight loss may indeed have contributed to a lower need for GLD treatment and, consequently, Zanders et al [1] should have used the words 'medication use' instead of 'medication adherence' in the title and conclusions of their paper.

Second, the authors propose in their discussion that stress following a major life event (such as stage IV cancer) could have contributed to decreased adherence. This proposal deserves some further attention. Chronic stress after cancer diagnosis can contribute to the development of major depression [5], which is relatively common in patients with cancer or diabetes $[6,7]$. Depression symptoms include decreased concentration, poor memory, loss of appetite, weight loss, pessimism, loss of interest and energy loss. It is conceivable that these symptoms hamper adequate diabetes self-care and contribute to poor health outcomes. Two systematic reviews indeed confirmed that depression is associated with treatment non-adherence [8] and higher mortality rates in type 2 diabetes [9].

Hence, though I fully agree with the conclusion of Zanders et al that the reason for the decline in the use of GLDs needs to be further elucidated among the different cancer types, I want to emphasise that a change in appetite, gastric discomfort, weight loss and chronic stress and depression should not be overlooked in these future studies. 
Duality of interest The author declares that there is no duality of interest associated with this manuscript.

Contribution statement The author was the sole contributor to this paper.

\section{References}

1. Zanders MM, Haak HR, van Herk-Sukel MPP, van de Poll-Franse LV, Johnson JA (2015) Impact of cancer on adherence to glucose-lowering drug treatment in individuals with diabetes. Diabetologia. doi:10.1007/ s00125-015-3497-8

2. American Society of Clinical Oncology (2014) Weight loss. Available from www.cancer.net/navigating-cancer-care/side-effects/weight-loss, accessed 11 Feb 2015

3. Lim EL, Hollingsworth KG, Aribisala BS, Chen MJ, Mathers JC, Taylor R (2011) Reversal of type 2 diabetes: normalisation of beta cell function in association with decreased pancreas and liver triacylglycerol. Diabetologia 54:2506-2514

4. Nicolini A, Ferrari P, Masoni MC et al (2013) Malnutrition, anorexia and cachexia in cancer patients: a mini-review on pathogenesis and treatment. Biomed Pharmacother 67:807-817

5. Pizzagalli DA (2014) Depression, stress, and anhedonia: toward a synthesis and integrated model. Annu Rev Clin Psychol 10:393-423

6. Ng CG, Boks MP, Zainal NZ, de Wit NJ (2011) The prevalence and pharmacotherapy of depression in cancer patients. J Affect Disord 131: $1-7$

7. Pouwer F, Nefs G, Nouwen A (2013) Adverse effects of depression on glycemic control and health outcomes in people with diabetes: a review. Endocrinol Metab Clin North Am 42:529-544

8. Gonzalez JS, Peyrot M, McCarl LA et al (2008) Depression and diabetes treatment nonadherence: a meta-analysis. Diabetes Care 31: 2398-2403

9. Van Dooren FE, Nefs G, Schram MT, Verhey FR, Denollet J, Pouwer F (2013) Depression and risk of mortality in people with diabetes mellitus: a systematic review and meta-analysis. PLoS One 8:e57058 